\title{
Robotic Setup Promises Consistent Effects of Multilocular Gastrointestinal Electrical Stimulation: First Results of a Porcine Study
}

\author{
Jonas F. Schiemer ${ }^{a} \quad K^{2}$ aren Stumm ${ }^{d}$ Karin H. Somerlik-Fuchs ${ }^{b}$ \\ Klaus-Peter Hoffmann ${ }^{c}$ Jan Baumgart ${ }^{d}$ Werner Kneist ${ }^{\text {a, e }}$ \\ a Department of General, Visceral, and Transplant Surgery, University Medical Center of \\ the Johannes Gutenberg University Mainz, Mainz, Germany; ${ }^{b}$ Department of Research \\ and Development, inomed Medizintechnik $\mathrm{GmbH}$, Emmendingen, Germany; ${ }^{\circ}$ Department \\ of Biomedical Engineering, Fraunhofer Institute for Biomedical Engineering, St. Ingbert, \\ Germany; ${ }^{d}$ Translational Animal Research Center, University Medical Center of the Johannes

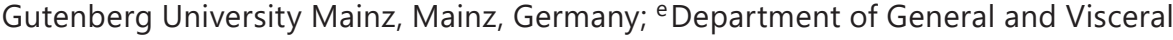 \\ Surgery, St. Georg Hospital Eisenach gGmbH, Eisenach, Germany
}

\section{Keywords}

Gastrointestinal motility · Motility disorder · Electromyography · Robotic surgical procedure

\begin{abstract}
Background: Electrical stimulation (ES) of several gastrointestinal (GI) segments is a promising therapeutic option for multilocular Gl dysmotility, but conventional surgical access by laparotomy involves a high degree of tissue trauma. We evaluated a minimally invasive surgical approach using a robotic surgical system to perform electromyographic (EMG) recordings and ES of several porcine GI segments, comparing these data to an open surgical approach by laparotomy. Materials and Methods: In 5 acute porcine experiments, we placed multiple electrodes on the stomach, duodenum, jejunum, ileum, and colon. Three experiments were performed with a median laparotomy and 2 others using a robotic platform. Multichannel EMGs were recorded, and ES was sequentially delivered with 4 ES parameters to the 5 target segments. We calculated pre- and poststimulatory spikes per minute (Spm) and performed a statistical Poisson analysis. Results: Electrode placement was achieved in all cases without complications. Increased technical and implantation time were required to achieve the robotic electrode placement, but invasiveness was markedly reduced in comparison to the conventional approach. The highest calculated (c)Spm values were found in the poststimulatory period of the small bowel with both the conventional and robotic approaches. Six of the 20 Poisson test results in the open setup reached statistical significance and 12 were significant in the robotic experiments. Conclusions: The robotic setup was less invasive, revealed more consistent effects of multilocular ES in several Gl segments, and is a promising option for future preclinical and clinical studies of GI motility disorders.




\section{Introduction}

The motility of the gastrointestinal (GI) tract is based on coordinated, smooth muscle contractions and relaxations, known as peristalsis. Motility irregularities can manifest throughout the entire GI tract, and they cause common, clinically relevant disorders. These disorders lead to significant impairment of the patient's quality of life [1]. Dysmotility symptoms range from dysphagia and gastroparesis to diarrhea, constipation, or even lifethreatening events in patients with chronic intestinal pseudo-obstruction [2]. Although the pathophysiology of motility disorders is not fully understood, impaired GI electrical activity is frequently correlated [3-8]. Studies have shown that GI electrical activity is measurable with smooth-muscle electromyography (EMG) and that GI motility can be modified with electrical stimulation (ES) [9-14]. Thus, a multilocular ES of the GI tract may be a promising midor long-term therapy for medically refractory GI motility disorders. However, to date, few studies have investigated the effects of multilocular ES in multiple segments of the GI tract in animals or humans [12-14]. We recently described successful modulation of electrical activity in 5 GI segments using variable ES [15]. The conventional open surgical approach to multiple GI segments still involves a laparotomy with increased tissue trauma and may impair accuracy in the detection of modulating effects from ES. Minimally invasive surgery may allow more realistic physiologic or pathophysiologic intraoperative GI electrical recordings.

The aim of our study was to establish a modified experimental protocol for robotic placement of electrodes for EMG and variable ES in multiple segments of the GI tract and to compare the technical and electrophysiological data for this promising minimally invasive approach to the conventional open surgery setup.

\section{Materials and Methods}

\section{Anesthesia and Surgery}

We performed acute experiments with 5 healthy, overnight-fasted male pigs weighing $28-46 \mathrm{~kg}$ (median $36 \mathrm{~kg}$ ) under general anesthesia and continuous monitoring. Three consecutive pigs underwent a conventional open procedure with midline laparotomy and placement of multiple monopolar hook-needle electrodes (inomed Medizintechnik GmbH, Emmendingen, Germany) in the stomach, duodenum, jejunum, ileum, and colon under general anesthesia (sodium thiopental 8-10 mg/ kg body weight $/ \mathrm{h}[\mathrm{mg} / \mathrm{kg} / \mathrm{h}]$ ) and piritramide $(0.3-0.4 \mathrm{mg} / \mathrm{kg} / \mathrm{h})$ as previously described [16]. Two other pigs were subjected to a robotic-assisted procedure under general anesthesia with ketamine $(5-10 \mathrm{mg} / \mathrm{kg} / \mathrm{h})$ and midazolam $(0.25-0.5 \mathrm{mg} / \mathrm{kg} / \mathrm{h})$, adapted to heart rate and blood pressure. For this purpose, a periumbilical skin incision was made and a Veress needle inserted to install a capnopneumoperitoneum of $10 \mathrm{~mm} \mathrm{Hg}$. Four robotic ports (8 $\mathrm{mm}$ ) were placed in the middle abdomen for the robotic surgical system (da Vinci Xi, Intuitive Surgical, Sunnyvale, CA, USA). A suprapubic catheter was inserted through the apex of the urinary bladder under laparoscopic assistance. Docking of the robotic surgical system was completed and surgical positioning was performed with a connected operating table (TruSystem 7000 dV OR table, Trumpf Medical System GmbH und Co. KG, Saalfeld, Germany). Hook-needle electrodes were placed analogously to the open setup using a robotic needle driver and fenestrated grasper (Fig. 1). The third robotic instrument was used for retraction under control of the operating surgeon at the surgeon's console. For both the open and robotic procedures, neutral electrodes were placed into the abdominal wall.

\section{Electromyography and Electrical Stimulation}

Impedance measurements and differential multichannel baseline EMGs were measured with an ISIS Xpress system (inomed Medizintechnik $\mathrm{GmbH}$ ). By measuring the impedance of all electrodes before recording the baseline EMG, we avoided short circuits or inappropriate tissue positioning. When the impedance was $>10 \mathrm{k} \Omega$, the electrodes were repositioned before starting the EMG recordings. A baseline multichannel smooth-muscle EMG was recorded for $3 \mathrm{~min}$ before stepwise application of 4 ES parameters. Gastric ES (GES), duodenal ES (DES), jejunal ES (JES), ileal ES (IES), and colonic ES (CES) were successively 
Fig. 1. Hook-needle electrodes were placed seromuscular in the ileum using robotic instruments.

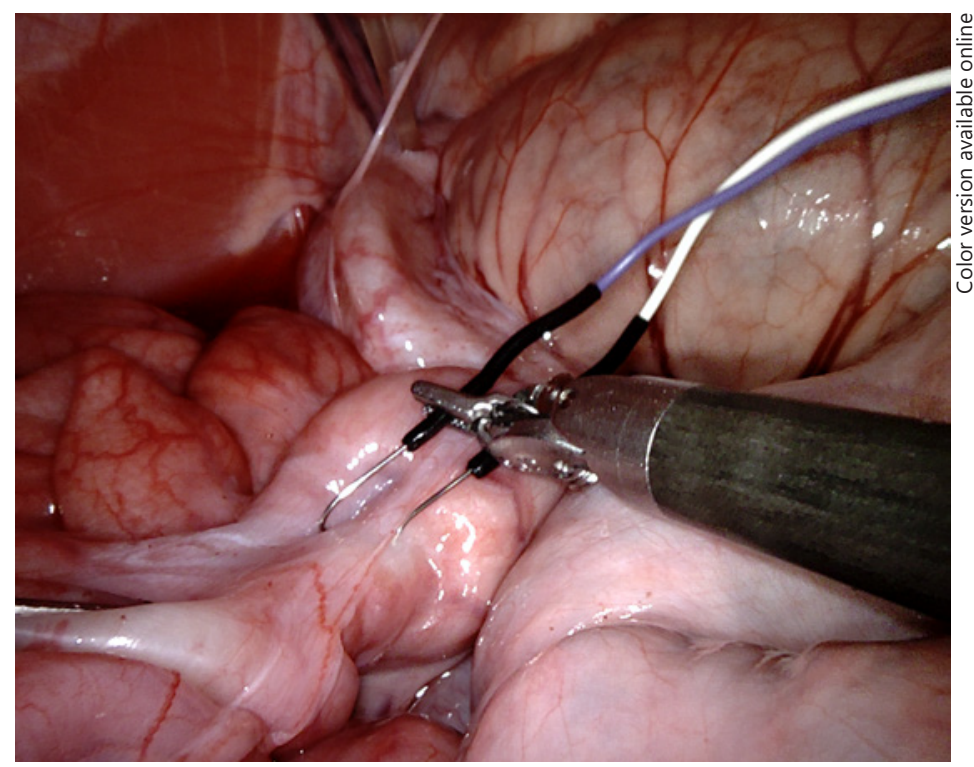

applied for $30 \mathrm{~s}$ of $25-\mathrm{mA}$ rectangular pulses. The open setup allowed pulse widths of $500 \mu \mathrm{s}$ (short pulse) or $1,000 \mu$ s (long pulse), and frequencies of $30 \mathrm{~Hz}$ (low frequency) or $130 \mathrm{~Hz}$ (high frequency). The ES parameters of the robotic approach were slightly modified, at pulse widths of 500 or $800 \mu \mathrm{s}$, respectively, and frequencies of 30 or $125 \mathrm{~Hz}$, respectively. After each ES administration, a poststimulatory multichannel smooth-muscle EMG was recorded for $3 \mathrm{~min}$. Eventually, animals were sacrificed with an intravenous injection of $20 \mathrm{~mL}$ of $7.45 \% \mathrm{KCl}$.

\section{Computer-Assisted Counting and Statistical Analysis}

The pre- and poststimulatory EMG analysis, spike detection, and statistical analysis were based on our previous study [15]. Minute-by-minute EMG spike activity was analyzed using data at 5 locations from 5 experiments $(n=25)$. For all experimental data, EMGs were $30-\mathrm{Hz}$ high-pass-filtered. Spikes were detected by setting a threshold normalizing spikes under the nonstimulation condition (resting spikes) to a mean of maximally 6 spikes per minute (Spm). Spikes under the next ES condition were algorithmically detected by using the resting spike threshold. To examine the effects of ES on the poststimulatory spike activity, we used an estimation of generalized linear mixed models (glmm) with multivariate normal random effects, with a penalized quasi-likelihood (PQL) method based on a penalized mixed Poisson model with penalization for overdispersion (function glmmPQL in package MASS of $R$ software v3.4.4; $R$ Core Team, 2018, R Foundation for Statistical Computing, Vienna, Austria). The statistical Poisson analysis was adapted for correlation within animals and multiple electrodes at the same location. This type of model is estimated for each location, each ES parameter set, and each of the 3 poststimulatory minutes. From the pooled experimental data, the glmmPQL function delivered an algorithmically estimated pre- and poststimulatory spike count, referred to here as calculated Spm (cSpm).

\section{Results}

\section{Surgical Procedure}

During all 5 acute experiments, there were no anesthesia-associated complications. The skin openings for the primary preparation required a 10-fold-larger incision for the open technique than for the robotic approach. All recording sites could be accessed with both the conventional and minimally invasive approaches. In both surgical approaches, all hookneedle electrodes were safely placed in the stomach, duodenum, jejunum, ileum, and colon without perforation, leakage, or relevant bleeding. The overall procedure duration of the 


\section{European \\ Surgical Research}

\begin{tabular}{l|l}
\hline Eur Surg Res 2020;61:14-22 \\
\hline DOI: 10.1159/000506799 & $\begin{array}{l}\text { @ 2020 The Author(s). Published by S. Karger AG, Basel } \\
\text { www.karger.com/esr }\end{array}$ \\
\hline
\end{tabular}

Schiemer et al.: Robotic Setup for Consistent Effects of Multilocular ES of the GI Tract

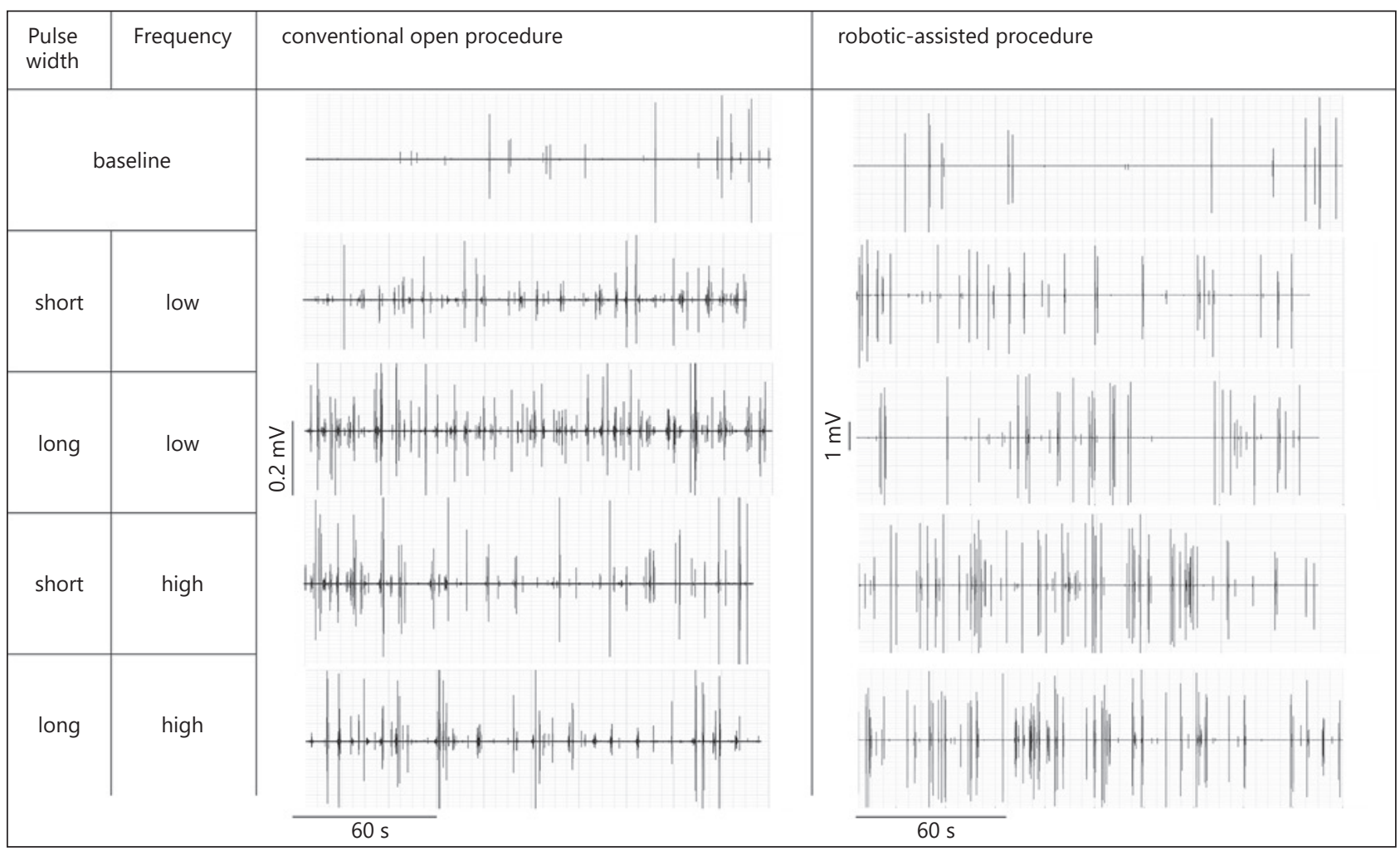

Fig. 2. Example for baseline, pre- and poststimulatory, multichannel, 30-Hz high-pass-filtered EMG recordings of an ileum segment. Electrical stimulations were performed with all 4 technical parameter sets.

Table 1. Relevant differences between conventional open surgery and the minimally invasive robotic approach

\begin{tabular}{lll}
\hline Parameters & Open surgical approach & Robotic approach \\
\hline Skin incision & approx. $400 \mathrm{~mm}$ & approx. $32 \mathrm{~mm}$ \\
Surrounding tissue trauma & high & low \\
Number of electrodes & Limited to serosal surface and & Limited to cable tangles and the \\
& electrode design & number of ports \\
Technical effort & low & high \\
Technology costs & low & high \\
Electrode handling & straightforward & straightforward \\
Procedure duration & $100 \%$ & $130 \%$ \\
Dehydration of serosal surfaces & high & low \\
\hline
\end{tabular}

robotic setup included port placement, docking, calibration, surgical exposure, and electrode placement, and it took approximately 30\% longer than the open setup with surgical access and electrode placement only. Shortly after the laparotomy, we visually observed a progressive dehydration of the serosal GI surfaces. In our minimally invasive technique, serosal moisture loss was not observed. In Table 1, we list the relevant differences between the conventional open and the minimally invasive robotic approach. 
Table 2. Algorithmically calculated spikes per minute and statistical Poisson analysis of 3 pre- and poststimulatory minutes for 5 gastrointestinal (GI) segments during conventional open and robotic porcine procedures

\begin{tabular}{|c|c|c|c|c|c|c|}
\hline \multirow[t]{2}{*}{ GI segment } & \multicolumn{2}{|l|}{ ES parameter } & \multicolumn{2}{|c|}{ Open approach } & \multicolumn{2}{|c|}{ Robotic approach } \\
\hline & pulse width & frequency & $\begin{array}{l}\text { cSpm } \\
\text { (3 min) }\end{array}$ & $p$ value & $\begin{array}{l}\text { cSpm } \\
\text { (3 min) }\end{array}$ & $p$ value \\
\hline \multirow[t]{5}{*}{ Stomach } & at baseline & & 5 & - & 2 & - \\
\hline & short & low & 9 & 0.051 & 68 & 0.201 \\
\hline & long & low & 10 & 0.038 & 11 & 0.565 \\
\hline & short & high & 10 & 0.028 & 10 & 0.596 \\
\hline & long & high & 10 & 0.033 & 7 & 0.698 \\
\hline \multirow[t]{5}{*}{ Duodenum } & at baseline & & 5 & - & 4 & - \\
\hline & short & low & 8 & 0.355 & 16 & 0.009 \\
\hline & long & low & 9 & 0.197 & 16 & 0.011 \\
\hline & short & high & 8 & 0.256 & 21 & 0.002 \\
\hline & long & high & 5 & 0.854 & 17 & 0.007 \\
\hline \multirow[t]{5}{*}{ Jejunum } & at baseline & & 1 & - & 2 & - \\
\hline & short & low & 111 & 0.081 & 42 & $<0.001$ \\
\hline & long & low & 127 & 0.073 & 38 & $<0.001$ \\
\hline & short & high & 100 & 0.089 & 47 & $<0.001$ \\
\hline & long & high & 106 & 0.085 & 32 & 0.001 \\
\hline \multirow[t]{5}{*}{ Ileum } & at baseline & & 3 & - & 1 & - \\
\hline & short & low & 10 & 0.076 & 158 & 0.012 \\
\hline & long & low & 20 & 0.004 & 165 & 0.011 \\
\hline & short & high & 18 & 0.007 & 211 & 0.008 \\
\hline & long & high & 17 & 0.008 & 159 & 0.012 \\
\hline \multirow[t]{5}{*}{ Colon } & at baseline & & 6 & - & 1 & - \\
\hline & short & low & 6 & 0.941 & 104 & 0.053 \\
\hline & long & low & 4 & 0.241 & 110 & 0.052 \\
\hline & short & high & 5 & 0.412 & 99 & 0.055 \\
\hline & long & high & 6 & 0.653 & 98 & 0.055 \\
\hline
\end{tabular}

During 5 acute experiments, we applied multiple hook-needle electrodes in the stomach, duodenum, jejunum, ileum, and colon of pigs. Baseline and poststimulatory EMGs were recorded, and electrical stimulation (ES) was performed with 4 technical parameter sets with a short pulse ( $500 \mu \mathrm{s})$, long pulse (800$1,000 \mu \mathrm{s})$, low frequency $(30 \mathrm{~Hz})$, or high frequency $(125-130 \mathrm{~Hz})$. Calculated spikes per minute (cSpm) and Poisson $p$ values for comparison of the 3 pre- and poststimulatory minutes were algorithmically determined (see Methods). Bold type indicates significant results $(p<0.05)$.

\section{Different Electrical Excitabilities}

Four ES parameters were successfully applied to all 5 target GI segments. In all 5 experiments, the pre- and poststimulatory multichannel EMGs were recorded (Fig. 2) and further analyzed. Table 2 shows the results of a detailed EMG analysis with cSpm and corresponding Poisson $p$ values for the comparison of the baseline and post-stimulatory cSpm. The highest cSpm of the open procedures was calculated for the poststimulatory EMGs of the jejunum after JES with a long pulse width and low frequency $(127 \mathrm{cSpm})$. The lowest cSpm was registered after CES with a long pulse width and low frequency in the 3 poststimulatory minutes of the colon $(4 \mathrm{cSpm})$. In the open experiments, most poststimulatory reactions after GES and IES were statistically distinct, whereas the comparison of pre- and poststimulatory cSpm of the duodenum, jejunum, and colon was statistically insignificant in the Poisson analysis. 
Overall, 6 of 20 test results in the open setup were statistically significant in comparison to the prestimulatory baseline.

EMG analysis of the robotic approach revealed the highest cSpm in the poststimulatory EMGs of the ileum after IES with a short pulse width and high frequency $(211 \mathrm{cSpm})$. The lowest cSpm was observed after GES with a long pulse width and high frequency $(21 \mathrm{cSpm})$. All cSpm responses after robotic DES, JES, and IES were statistically distinct in the Poisson analysis. The test results of the poststimulatory gastric and colonic EMG after robotic GES and CES remained insignificant in the Poisson analysis. Overall, 12 of 20 Poisson test results in the robotic setup were statistically significant.

\section{Discussion}

In this experimental study, we established a robotic setup for the placement of EMG and ES electrodes in 5 segments of the porcine GI tract, and then compared the surgical and electrophysiological results of this comfortable and minimally invasive access to our conventional surgical approach. Tremor filtering, motion scaling, and instruments with $7 \mathrm{df}$ make the robotic variant appear superior to the laparoscopic one. To the best of our knowledge, no previous study has involved the assessment of a robotic approach for variable ES and EMG measurements in 5 segments of the porcine GI tract.

The robotic procedure required a more technical setup, but the skin incisions and surrounding tissue trauma were minimal in comparison to the open procedure. Robotic exposure of the target GI segment was excellent, and electrode placement with the 3 robotic instruments was easy, although the electrode cables caused some interference. The robotic procedure appeared to take longer than the open experiments because docking and calibration are mandatory, and surgical exposure is performed with the robotic instruments and the connected operating table. Nevertheless, the minimally invasive robotic approach offers the potential advantages of achieving more realistic physiologic or pathophysiologic intraoperative GI electrical recordings. Throughout the robotic procedures, serosal dehydration was negligible in comparison to the conventional setup. This difference can be explained by the porcine abdominal cavity having no continuous connection to the relatively dry and cold air of the operating theatre, and convection was regarded as being minimal.

It seems reasonable to assume that a less physiological environment alters GI electrical signals. The electrophysiological consequences of GI serosal dehydration have not previously been investigated, and published studies are few. A clinical study described delayed gastric emptying in systemically dehydrated healthy participants [17]. Other studies with healthy participants and patients with dysmotility indicated that the human GI tract significantly responds to alterations in temperature $[18,19]$.

Our electrophysiological analysis provided evidence of a strong and reliable spike alteration through the application of ES in multiple segments of the GI tract. After multilocular ES of 5 GI segments, automated EMG spike calculation within an early poststimulatory period revealed relevant cSpm changes in all investigated GI parts of the 5 experiments. In both the open and robotic experiments, several cSpm measures of the 3 poststimulatory minutes were markedly higher than the prestimulatory baseline EMG in the stomach, duodenum, jejunum, and ileum. Only the pre- and poststimulatory cSpm of the colon in the open experiments were comparably low. In contrast, EMG analysis of the colon during the robotic experiments revealed very high but statistically insignificant increases in cSpm compared to baseline. The highest spike counts were found in the small bowel of both the conventional (jejunum) and robotic (ileum) setup. This result is consistent with our previous findings [15] and other studies. Romański et al. [20] and Lammers et al. [21] reported a higher number of Spm in the 
jejunum and ileum of pigs and dogs than in the human stomach and duodenum [22] and the porcine colon [23].

The statistical group analysis revealed that the effects of ES were more consistent in the robotic setup. Overall, there were twice as many statistically significant Poisson test results with the robotic approach than with the conventional setup. However, the electrophysiological data were likely influenced by the different surgical approaches and anesthesiologic regimens and energy-reduced ES parameters in the robotic approach.

The general anesthesia of the conventional experiments was maintained with thiopental and piritramide, and the robotic experiments were performed with ketamine and midazolam. Published findings on the effects of our anesthetic regimens on GI motility are mixed and controversial. Thiopental has been described as conserving a normal migrating myoelectrical complex in the small intestine of rats [24]. A canine study of conventional surgical access by laparotomy found that thiopental even triggered spike activity in the duodenum and jejunum but not in the ileum or stomach. The same study found no effects of ketamine on the GI spike activity [25]. Inhibition of the entire GI transit is induced by the administration of opioid drugs [26] like piritramide, and reduced spike activity has been described in the stomach and colon of morphinetreated rats [27]. Although midazolam shows only subtle effects in gastric, duodenal, and jejunal manometry of healthy volunteers [28], significant inhibition of GI transit has been found in mice [29].

GI electrical signals may also be influenced by the physiologic milieu. This includes blood pressure, heart rate, oxygenation, and body temperature. Under general anesthesia, these factors were kept within homeostatic ranges. Evidence of the direct influence of the surgical access on GI electrical activity is limited. Laparotomy with incision of the muscle layers and peritoneum has been described as having an inhibitory effect on GI spike activity in rats [24]. On the other hand, a faster colonic transit was found in patients after minimally invasive surgery compared to open surgery [30].

Faster recovery from postoperative intestinal paralysis was also found after laparoscopic surgery compared to conventional surgery in a canine study [31]. The electrical stimulation parameters of the robotic setup had to be slightly reduced in comparison to the open approach to achieve cross-platform compatibility of our electrotechnical setup and the robotic surgical system. During the ES in the robotic experiments, we applied pulse widths of $800 \mu$ s instead of $1,000 \mu \mathrm{s}$ and frequencies of $125 \mathrm{~Hz}$ instead of $130 \mathrm{~Hz}$. All applied ES parameters were in the range of the available literature and the pulse width was, comparably, very low $[5,9$, $11-13,15,16,32,33]$.

In conclusion, we have demonstrated several advantages of the minimally invasive robotic setup in comparison to the more invasive open surgery approach. The robotic setup appears to be the more high-grade and promising method for clinical application; clinical realization of the open approach would likely involve disproportionately high morbidity and complications. A case report positively evaluated the robotic surgical system in the placement of a GES system for a patient with medically refractory gastroparesis [34]. Moreover, the robotic surgical system was able to easily access the stomach, duodenum, jejunum, ileum, and colon investigated in this study, but also the rectum and esophagus via small skin incisions. Although we report on an experimental study, its clinical application in patients with medically refractory GI motility disorders for electrical activity mapping of several GI segments would already be technically feasible with our minimally invasive setup. Furthermore, a first individual motility evaluation would take place in a more physiological environment. Together with recent advances in wireless GI device developments [33, 35, 36], even the scenario of robotic placement of multiple theranostic devices distributed along the GI tract appears reasonable.

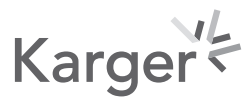




\section{Acknowledgments}

We thank Hubert Stein (Intuitive Surgical, Sunnyvale, CA, USA) and Dr. Heiko Ziervogel and his team (Medical Center of Excellence c/o HCx Consulting GmbH, Wendisch Rietz, Germany) for experimental assistance; Dr. Thilo Krüger and Kornelius Lente (Department of Research and Development, inomed Medizintechnik GmbH, Emmendingen, Germany) for electrotechnical and spike calculation support; and Prof. Dr. Manfred Berres (Institute of Medical Biostatistics, Epidemiology and Informatics, University Medical Center of the Johannes Gutenberg-University Mainz, Germany and RheinAhrCampus, University of Applied Sciences Koblenz, Remagen, Germany) for help with statistics. We also appreciate the support of Dr. Nadine Baumgart and Jana Dickmann (Translational Animal Research Center, University Medical Center of the Johannes Gutenberg-University Mainz, Germany), Dr. Axel Heimann and Prof. Dr. Oliver Kempski (Institute for Neurosurgical Pathophysiology, University Medicine of the Johannes Gutenberg-University Mainz, Germany), and Roman Ruff (Department of Biomedical Engineering, Fraunhofer Institute for Biomedical Engineering, St. Ingbert, Germany).

\section{Statement of Ethics}

This study complied with all relevant national regulations and institutional policies for the care and use of animals. It was approved by the Regional Board of Animal Welfare, Koblenz, Rhineland-Palatinate, Germany, under approval code G-17-1-008.

\section{Conflict of Interest Statement}

J.F.S., K.S., K.P.H., J.B., and W.K. declare no conflicts of interest. K.H.S. is an employee of inomed Medizintechnik GmbH.

\section{Funding Sources}

This study was part of the INTAKT project and funded by the Federal Ministry of Education and Research (BMBF, grant No. 16SV7638).

\section{Author Contributions}

W.K. and K.P.H. obtained funding. J.F.S., K.S., K.H.S.F., and W.K. were involved in the study design and experiments. J.B. handled laboratory animal issues. J.F.S. and W.K. analyzed and interpreted the data and wrote the manuscript.

\section{References}

1 Lacy BE, Weiser K. Gastrointestinal motility disorders: an update. Dig Dis. 2006;24(3-4):228-42.

2 Connor FL, Di Lorenzo C. Chronic intestinal pseudo-obstruction: assessment and management. Gastroenterology. 2006 Feb;130(2 Suppl 1):S29-36.

3 Lammers WJ. Arrhythmias in the gut. Neurogastroenterol Motil. 2013 May;25(5):353-7.

4 Martellucci J, Valeri A. Colonic electrical stimulation for the treatment of slow-transit constipation: a preliminary pilot study. Surg Endosc. 2014 Feb;28(2):691-7.

5 Shafik A, Shafik AA, El-Sibai O, Ahmed I. Colonic pacing: a therapeutic option for the treatment of constipation due to total colonic inertia. Arch Surg. 2004 Jul;139(7):775-9.

6 Lin Z, Eaker EY, Sarosiek I, McCallum RW. Gastric myoelectrical activity and gastric emptying in patients with functional dyspepsia. Am J Gastroenterol. 1999 Sep;94(9):2384-9.

7 Angeli TR, Du P, Midgley D, Paskaranandavadivel N, Sathar S, Lahr C, et al. Acute slow wave responses to highfrequency gastric electrical stimulation in patients with gastroparesis defined by high-resolution mapping. Neuromodulation. 2016 Dec;19(8):864-71. 
8 O'Grady G, Wang TH, Du P, Angeli T, Lammers WJ, Cheng LK. Recent progress in gastric arrhythmia: pathophysiology, clinical significance and future horizons. Clin Exp Pharmacol Physiol. 2014 Oct;41(10):854-62.

9 Chen JD, Yin J, Wei W. Electrical therapies for gastrointestinal motility disorders. Expert Rev Gastroenterol Hepatol. 2017 May;11(5):407-18.

10 Avalos DJ, Sarosiek I, Loganathan P, McCallum RW. Diabetic gastroparesis: current challenges and future prospects. Clin Exp Gastroenterol. 2018 Sep;11:347-63.

11 Liu S, Hou X, Chen JD. Therapeutic potential of duodenal electrical stimulation for obesity: acute effects on gastric emptying and water intake. Am J Gastroenterol. 2005 Apr;100(4):792-6.

12 Sun Y, Song GQ, Yin J, Lei Y, Chen JD. Effects and mechanisms of gastrointestinal electrical stimulation on slow waves: a systematic canine study. Am J Physiol Regul Integr Comp Physiol. 2009 Nov;297(5):R1392-9.

$13 \mathrm{Xu} \mathrm{X,} \mathrm{Lei} \mathrm{Y,} \mathrm{Chen} \mathrm{JD.} \mathrm{Effects} \mathrm{and} \mathrm{mechanisms} \mathrm{of} \mathrm{electrical} \mathrm{stimulation} \mathrm{of} \mathrm{the} \mathrm{stomach,} \mathrm{duodenum,} \mathrm{ileum,} \mathrm{and}$ colon on gastric tone in dogs. Dig Dis Sci. 2010 Apr;55(4):895-901.

14 Agrawal A, Francis SL, Deveneau NE, Jain S, Abrasley C, McNeese JT, et al. Gastric electrical stimulation and sacral electrical stimulation: A long-term follow-up study of dual-device treatment. Dig Dis Sci. 2016 Jan; 61(1):176-80

15 Schiemer JF, Heimann A, Somerlik-Fuchs KH, Ruff R, Hoffmann KP, Baumgart J, et al. Five-fold gastrointestinal electrical stimulation with EMG-based activity analysis: towards mul-tilocular theranostic intestinal implants. J Neurogastroenterol Motil. 2019 Jul;25(3):461-70.

16 Schiemer JF, Heimann A, Somerlik-Fuchs KH, Ruff R, Hoffmann KP, Baumgart J, et al. Electrical stimulation with motility analysis of five parts of the gastrointestinal tract. Curr Dir Biomed Eng. 2018;4(1):9-11.

17 van Nieuwenhoven MA, Vriens BE, Brummer RJ, Brouns F. Effect of dehydration on gastrointestinal function at rest and during exercise in humans. Eur J Appl Physiol. 2000 Dec;83(6):578-84.

18 Wang RF, Wang ZF, Ke MY, Fang XC, Sun XH, Zhu LM, et al. Temperature can influence gastric accommodation and sensitivity in functional dyspepsia with epigastric pain syndrome. Dig Dis Sci. 2013 Sep;58(9):2550-5.

19 Shafik A, Shafik I, El Sibai O, Shafik AA. Rectal cooling test in the differentiation between constipation due to rectal inertia and anismus. Tech Coloproctol. 2007 Mar;11(1):39-43.

20 Romański KW, Rudnicki J, Sławuta P. The myoelectric activity of ileum in fasted and fed young pigs. J Physiol Pharmacol. 2001 Dec;52(4 Pt 2):851-61.

21 Lammers WJ, Donck LV, Schuurkes JA, Stephen B. Longitudinal and circumferential spike patches in the canine small intestine in vivo. Am J Physiol Gastrointest Liver Physiol. 2003 Nov;285(5):G1014-27.

22 Lychkova AE. Pacemakers of the upper divisions of the digestive tract. Bull Exp Biol Med. 2014 Feb;156(4): 518-21.

23 Shafik A, El-Sibai O, Ahmed A. Study of the mechanism underlying the difference in mo-tility between the large and small intestine: the "single" and "multiple" pacemaker theory. Front Biosci. 2001;6(2):1-5.

24 Bueno L, Ferre JP, Ruckebusch Y. Effects of anesthesia and surgical procedures on intestinal myoelectric activity in rats. Am J Dig Dis. 1978 Aug;23(8):690-5.

25 Healy TE, Foster GE, Evans DF, Syed A. Effect of some i.v. anaesthetic agents on canine gastrointestinal motility. Br J Anaesth. 1981 Mar;53(3):229-33.

26 Manara L, Bianchetti A. The central and peripheral influences of opioids on gastrointestinal propulsion. Annu Rev Pharmacol Toxicol. 1985;25(1):249-73.

27 Primi MP, Bueno L. Effects of centrally administered naloxone on gastrointestinal myoelectrical activity in morphine-dependent rats. J Pharmacol Exp Ther. 1987 Jan;240(1):320-6.

28 Castedal M, Björnsson E, Abrahamsson H. Effects of midazolam on small bowel motility in humans. Aliment Pharmacol Ther. 2000 May;14(5):571-7.

29 Inada T, Asai T, Yamada M, Shingu K. Propofol and midazolam inhibit gastric emptying and gastrointestinal transit in mice. Anesth Analg. 2004 Oct;99(4):1102-6.

30 van Bree SH, Vlug MS, Bemelman WA, Hollmann MW, Ubbink DT, Zwinderman AH, et al. Faster recovery of gastrointestinal transit after laparoscopy and fast-track care in patients undergoing colonic surgery. Gastroenterology. 2011 Sep;141(3):872-880.e1.

31 Böhm B, Milsom JW, Fazio VW. Postoperative intestinal motility following conventional and laparoscopic intestinal surgery. Arch Surg. 1995 Apr;130(4):415-9.

32 Aellen S, Wiesel PH, Gardaz JP, Schlageter V, Bertschi M, Virag N, et al. Electrical stimulation induces propagated colonic contractions in an experimental model. Br J Surg. 2009 Feb;96(2):214-20.

33 Lo YK, Wang PM, Dubrovsky G, Wu MD, Chan M, Dunn JC, et al. Wireless implant for gastrointestinal motility disorders. Micromachines (Basel). 2018 Jan;9(1):17.

34 Saurabh S, Lee GO, Chong HS. Robotic gastric electrical stimulator placement: technique and literature review. J Robot Surg. 2014 Sep;8(3):285-7.

35 Wang R, Abukhalaf Z, Javan-Khoshkholgh A, Wang TH, Sathar S, Du P, et al. A Miniature Configurable Wireless System for Recording Gastric Electrophysiological Activity and Delivering High-Energy Electrical Stimulation. IEEE J Emerg Sel Top Circuits Syst. 2018 Jun;8(2):221-9.

36 Zhu B, Wang Y, Yan G, Jiang P, Liu Z. A gastrointestinal electrical stimulation system based on transcutaneous power transmission technology. Gastroenterol Res Pract. 2014;2014:728572. 